\title{
Isoperimetric inequalities \& volume comparison theorems on CR manifolds
}

\author{
Sagun Chanillo And PAUl C. YANG
}

\begin{abstract}
In this article we study the Jacobi equation associated with the geodesics in a pseudo-hermitian manifold wish vanishing Webster torsion. We develop integral geometric formula generalizing the well known Santalo formula in Riemannian geometry. As applications we obtain volume comparison results under suitable curvature assumptions as well as isoperimetric inequalities for domains in such manifolds.
\end{abstract}

Mathematics Subject Classification (2000): 32V20 (primary); 32V05, 53C17, $53 \mathrm{C} 21$ (secondary).

\section{Introduction}

In this paper we study a manifold $M^{3}$ with a contact structure $\Theta$ and a compatible CR-structure, that is an almost complex structure $J$ defined on the contact planes $\Xi$ which are given as the kernel of the contact form $\Theta$. In [7, 10], Webster and Tanaka introduced the pseudo-hermitian connection and the associated torsion and curvature tensors in solving the equivalence problem. This work provides an analytic frame work for study of the geometry of CR structures. We are interested in developing, along the lines of Riemannian geometry, volume comparison criteria as well as isoperimetric inequalities. A cursory examination of the equation of geodesics shows that it comprises a third order system, and hence quite difficult to study. In this paper we make an essential simplifying assumption, the vanishing of a certain component of torsion, that reduces the equation of geodesics to a second order system. Geometrically, the vanishing torsion condition means that the Reeb vector field is an infinitesimal CR transformation. The torsion free condition means that for each value of the geodesic curvature $\alpha$, there is a foliation of the unit contact bundle associated to the geodesic flow along geodesics of curvature $\alpha$. Thus it is possible to generalize the well known integral geometric formulae of Santalo to this setting.

Both S. C. and P. Y. were partially supported by NSF grants.

Received April 24, 2008; accepted September 3, 2008. 
For pseudo-hermitian structures satisfying this vanishing torsion conditon and having bounded Webster curvature we develop volume comparison results based on an ODE which expresses the volume element associated to an exponential map as a Wronskian. In addition, we introduce the notion of A-injectivity radius and derive a bound for it in terms of the diameter, volume and the curvature bound. This gives an analogue of Cheeger's bound for the injectivity radius in the Riemannian setting. For simplicity, we have stated these results in 3D, but it is clear that the argument works in higher dimensional setting.

Finally, we derive an isoperimetric inequality for domains in a 3D pseudohermitian structure of bounded Webster scalar curvature. The first result extends the well known inequality first given by Pansu [5] for the Heisenberg group to simply connected 3D pseudo-hermitian manifolds of non-positive Webster scalar curvature. This result uses the special feature of area minimizing surfaces in $3 \mathrm{D}$, hence does not generalize to higher dimension. A second result applies to compact pseudo-hermitian manifolds of positive Webster scalar curvature, this proof makes use of the generalized Santalo formula. This isoperimetric inequality does not yield the correct homogeneity that should be natural. We do not know at this time, whether this is due to the defect of the method, or it is an intrinsic feature. We impose a width condition to recover the expected isoperimetric inquality. As a consequence, we obtain a corresponding Sobolev inequality generalizing the work of Varopolous [9].

\section{The Jacobi equation on a CR manifold}

Given a contact form $\Theta$, it determines a contact plane $\Xi=\operatorname{Ker} \Theta$. Then there is a unique Reeb vector field $T$ determined by the conditions $\Theta(T)=1$ and $L_{T} \Theta=0$.

We recall the connection of Tanaka [7] and Webster [10]. We can then choose a complex vector field $Z_{1}$ to be an eigenvector of $J$ with eigenvalue $i$, and a complex 1 -form $\theta^{1}$ such that

$$
\left\{\Theta, \theta^{1}, \theta^{\overline{1}}\right\} \text { is dual to }\left\{T, Z_{1}, Z_{\overline{1}}\right\} .
$$

It follows that

$$
d \Theta=i h_{1 \overline{1}} \theta^{1} \wedge \theta^{\overline{1}} \text { for real } h_{1 \overline{1}}>0
$$

then can normalize further by choosing $Z_{1}$ so that $h_{1 \overline{1}}=1$

$$
d \Theta=i \theta^{1} \wedge \theta^{\overline{1}} .
$$

The pseudo-hermitian connection $\nabla$ is given by

$$
\nabla Z_{1}=\omega_{1}^{1} \otimes Z_{1}, \nabla Z_{\overline{1}}=\omega_{\overline{1}}^{\overline{1}} \otimes Z_{\overline{1}}, \nabla T=0 .
$$


The connection form $\omega_{1}^{1}$ is uniquely determined by

$$
\left\{\begin{array}{l}
d \theta^{1}=\theta^{1} \wedge \omega_{1}^{1}+A_{\overline{1}}^{1} \Theta \wedge \theta^{\overline{1}} \\
\omega_{1}^{1}+\omega_{\overline{1}}^{\overline{1}}=0 .
\end{array}\right.
$$

Then

$$
\begin{aligned}
& d \omega_{1}^{1}=R \theta^{1} \wedge \theta^{\overline{1}}+2 i \operatorname{Im}\left(A_{11, \overline{1}}\right) \theta^{\overline{1}} \wedge \Theta \text { where } \\
& A_{\overline{1}}^{1}-\text { Torsion } \\
& R-\text { Webster scalar curvature. }
\end{aligned}
$$

Converting to real forms: $\theta^{1}=e^{1}+\sqrt{-1} e^{2}, Z_{1}=\frac{1}{2}\left(e_{1}-i e_{2}\right), \omega_{1}^{1}=i w$

$$
\begin{aligned}
d \Theta & =2 e^{1} \wedge e^{2} \\
\nabla e_{1} & =\omega \otimes e_{2}, \quad \nabla e_{2}=-\omega \otimes e_{1} \\
d e^{1} & =-e^{2} \wedge \omega+\Theta \wedge\left(\Re A_{1}^{1} e^{1}+\Im A_{1}^{1} e^{2}\right) \\
d e^{2} & =e^{1} \wedge \omega+\Theta \wedge\left(\Im A_{1}^{1} e^{1}-\Re A_{1}^{1} e^{2}\right) . \\
d \omega\left(e_{1}, e_{2}\right) & =-2 R \\
{\left[e_{1}, e_{2}\right] } & =-2 T-\omega\left(e_{1}\right) e_{1}-\omega\left(e_{2}\right) e_{2} \\
{\left[e_{1}, T\right] } & =\left(\Re A_{11}\right) e_{1}-\left[\left(\Im A_{11}\right)+\omega(T)\right] e_{2} \\
{\left[e_{2}, T\right] } & =\left[\Im A_{11}+\omega(T)\right] e_{1}+\left(\Re A_{11}\right) e_{2} .
\end{aligned}
$$

Extend $J$ to all $T M$ by requiring $J(T)=0$ so that

$$
J^{2} x=-x+\Theta(x) T \quad \forall x \in T M .
$$

The condition on torsion we will assume in this paper is:

$$
\text { Tor }\left.(T, Y)\right|_{\Xi}=0 \text {. }
$$

The statement (1.1) is equivalent to the vanishing of $A_{11}$ and geometrically means $T$ is an infinitesimal CR transformation [10].

Now we proceed to describe the equation of geodesics.

Lemma 1.1 ([6]). The Geodesic equation under (1.1) is

$$
\nabla_{X} X=\alpha J X, X \alpha=-<\operatorname{Tor}(T, X), X>=0
$$

where $X$ is the unit tangent vector to the geodesic. 
Thus the vanishing torsion assumption implies that all geodesics have constant curvature $\alpha$ which may be regarded as a parameter. In addition, the vanishing torsion condition reduces the geodesic equation to a second order system. In analogy with the exponential map in Riemannian geometry, we parametrize a neighborhood of a point $p \in M$ by shooting out unit speed contact geodesics. Thus there will be two parameters associated with each geodesic issuing from $p$ : its curvature $\alpha$ and its initial directions in $\Xi_{p}$. We will need to consider two types of variation of geodesics. One type of variation is through the initial angle $\phi$ that our geodesic makes in the contact plane with a fixed direction. The variation vector in this direction will be denoted as $Y_{\phi}$.

Another variation will be via the curvature $\alpha$. The variation vector in this direction will be denoted by $Y_{\alpha}$. According to the calculations of Rumin [6], if the perturbed geodesic is to remain a Legendrian curve we need to have,

Lemma 1.2. For arc-length paramter $s$ along the unit speed geodesic, we have:

(a) $Y_{\phi}=\alpha(s) c_{\phi}(s) X+c_{\phi}^{\prime}(s) J X+c_{\phi}(s) T$

(b) $Y_{\alpha}=\alpha(s) c_{\alpha}(s) X+c_{\alpha}^{\prime}(s) J X+c_{\alpha}(s) T$.

Lemma 1.3. For a geodesic variation vector field given by,

$$
Y(s)=\alpha(s) c(s) X+c^{\prime}(s) J X+c(s) T
$$

we have,

(a) $Y^{\prime}(s)=\left(c^{\prime \prime}+\alpha^{2} c(s)\right) J X+c^{\prime}(s) T$

and,

(b) $Y^{\prime \prime}=\left(c^{\prime \prime}+\alpha^{2} c(s)\right)^{\prime} J X-\alpha\left(c^{\prime \prime}+\alpha^{2} c(s)\right) X+c^{\prime \prime}(s) T$.

We are now ready to derive the Jacobi equation.

Lemma 1.4. Let $Y$ denote a variation vector field that arises as a variation of the geodesic from a one parameter family of perturbations that maintain the perturbed curve in the contact plane. Let $\Xi$ denote the contact plane. Under the assumption (1.1) we have that the Jacobi equation for the variation vector field is,

$$
Y^{\prime \prime}+R(Y, X) X-\alpha J Y^{\prime}-\left.Y(\alpha) J X\right|_{\Xi}=0 .
$$

Proof. We start with the geodesic equation Lemma 1.1, and taking its covariant derivative in $Y$ we get,

$$
\nabla_{Y} \nabla_{X} X=Y(\alpha) J X+\alpha J \nabla_{Y} X .
$$

Now note,

$$
\nabla_{Y} X=\nabla_{X} Y-[X, Y]-\operatorname{Tor}(X, Y) .
$$

Now $\left[\frac{\partial}{\partial s}, \frac{\partial}{\partial \varphi}\right]=\left[\frac{\partial}{\partial s}, \frac{\partial}{\partial \alpha}\right]=0$ and so $[X, Y]=0$. 
Inserting (1.3) into the right side of (1.2) we get, using $[X, Y]=0$, and (1.1),

$$
J \nabla_{Y} X=J\left(\nabla_{X} Y-\Theta(\operatorname{Tor}(X, Y)) T\right) .
$$

But now $J T=0$, thus,

$$
J \nabla_{Y} X=J \nabla_{X} Y
$$

Using (1.4), (1.2) becomes,

$$
\nabla_{Y} \nabla_{X} X=Y(\alpha) J X+\alpha J \nabla_{X} Y .
$$

Since $J T=0$ notice the right side of (1.5) lies in $\Xi$. Now re-writing the left side of (1.5), we get,

$$
\begin{aligned}
\nabla_{Y} \nabla_{X} X & =\nabla_{Y} \nabla_{X} X-\nabla_{X} \nabla_{Y} X+\nabla_{X} \nabla_{Y} X \\
& =\nabla_{X} \nabla_{Y} X+R(Y, X) X .
\end{aligned}
$$

From (1.3) again, we may simplify the expression above,

$$
=\nabla_{X} \nabla_{Y} X+R(Y, X) X=\nabla_{X} \nabla_{X} Y+R(Y, X) X-\nabla_{X}([X, Y]+\operatorname{Tor}(X, Y)) \text {. }
$$

Now $[X, Y]=0$ and by (1.1) again,

$$
\text { Tor }(X, Y)=d \Theta(X, Y) T .
$$

Since $Y$ is a Legendrian variation we can write

$$
Y=\alpha c(s) X+c^{\prime}(s) J X+c(s) T .
$$

Substituting in (1.7) we get,

$$
\text { Tor }(X, Y)=d \Theta\left(X, \alpha c(s) X+c^{\prime}(s) J X+c(s) T\right) T .
$$

Hence,

$$
\operatorname{Tor}(X, Y)=d \Theta\left(X, c^{\prime}(s) J X\right) T=c^{\prime}(s) T .
$$

Thus (1.6) simplifies to,

$$
\nabla_{X} \nabla_{X} Y+R(Y, X) X-\nabla_{X}\left(c^{\prime}(s) T\right)=\nabla_{X} \nabla_{X} Y+R(Y, X) X-c^{\prime \prime}(s) T .
$$

Now by Lemma 1.3(b),

$$
\begin{aligned}
& \nabla_{X} \nabla_{X} Y=Y^{\prime \prime}=\left.Y^{\prime \prime}\right|_{\Xi}+c^{\prime \prime}(s) T \\
& \nabla_{Y} \nabla_{X} X=\left.Y^{\prime \prime}\right|_{\Xi}+R(Y, X) X .
\end{aligned}
$$

Now using (1.5) we finally get,

$$
\left.Y^{\prime \prime}\right|_{\Xi}+R(Y, X) X-Y(\alpha) J X-\alpha J \nabla_{X} Y=0 .
$$

Since we are assuming $(1.1), R(Y, X) X$ is contact and so this proves our lemma. 
Our next aim is to compute the ODE satisfied by the coefficients $c_{\phi}, c_{\alpha}$ of the variation fields $Y_{\phi}, Y_{\alpha}$ as defined in Lemma 1.2(a), (b). We have,

Lemma 1.5. Let us denote $\langle R(J X, X) X, J X\rangle=R(s)$. Then,

(a) $\left(c_{\phi}^{\prime \prime}+\alpha^{2} c_{\phi}\right)^{\prime}+R(s) c_{\phi}^{\prime}=0, c_{\phi}(0)=0, c_{\phi}^{\prime}(0)=0$ and

(b) $\left(c_{\alpha}^{\prime \prime}+\alpha^{2} c_{\alpha}\right)^{\prime}+R(s) c_{\alpha}^{\prime}=1, c_{\alpha}(0)=0, c_{\alpha}^{\prime}(0)=0$.

Proof. We use Lemma 1.4 in conjunction with Lemma 1.3. To prove Lemma 1.5(a), first notice that $Y_{\phi}=\nabla_{\frac{\partial}{\partial \phi}}$. Thus, $Y_{\phi}(\alpha)=0$. Thus our Jacobi equation reads,

$$
Y_{\phi}^{\prime \prime}+c_{\phi}^{\prime}(s) R(J X, X) X-\alpha J Y_{\phi}^{\prime} \mid \Xi=0 .
$$

Using the expressions from Lemma 3 for $Y_{\phi}^{\prime}$ and $Y_{\phi}^{\prime \prime}$ and inserting into the expression above, after simplification we have,

$$
\left[\left(c_{\phi}^{\prime \prime}(s)+\alpha^{2} c_{\phi}(s)\right)^{\prime}+R(s) c_{\phi}^{\prime}(s)\right] J X=0 .
$$

The initial conditions on $c_{\phi}$ follow from the demand that $Y_{\phi}(0)=0$ when applied to Lemma 1.2. Thus, we immediately get Lemma 1.5(a).

We now obtain Lemma 1.5(b). Since $Y_{\alpha}=\nabla_{\frac{\partial}{\partial \alpha}}$, it follows $Y_{\alpha}(\alpha)=1$. Thus our Jacobi equation is now,

$$
Y_{\alpha}^{\prime \prime}+c_{\alpha}^{\prime}(s) R(J X, X) X-J X-\left.\alpha J Y_{\alpha}^{\prime}\right|_{\Xi}=0 .
$$

Using Lemma 1.3(b) again in the expression above and simplifying we get,

$$
\left[\left(c_{\alpha}^{\prime \prime}(s)+\alpha^{2} c_{\alpha}(s)\right)^{\prime}+R(s) c_{\alpha}^{\prime}(s)-1\right] J X=0 .
$$

The initial conditions on $c_{\alpha}$ follows from the demand that $Y_{\alpha}(0)=0$. This immediately gives us Lemma 1.5(b).

Our next goal is to compute an ODE for the Jacobian density of the volume form. It will turn out to be a Wronskian. We have,

Lemma 1.6. For the Wronskian,

$$
W(s, \phi, \alpha)=\Theta \wedge d \Theta\left(X, Y_{\phi}, Y_{\alpha}\right)=\Theta\left(Y_{\phi}\right) \Theta\left(Y_{\alpha}^{\prime}\right)-\Theta\left(Y_{\alpha}\right) \Theta\left(Y_{\phi}^{\prime}\right)
$$

we have,

$$
W^{\prime \prime}+\left(\alpha^{2}+R(s)\right) W=2 c_{\phi}, W(0)=0, W^{\prime}(0)=0 .
$$

Proof. The right side of the identity above follows because of Cartan's identity and use of the variation formulae for $Y_{\phi}$ and $Y_{\alpha}$ in Lemma 1.2(a), (b) and the fact $\Theta(X)=0$. Differentiating $W$ twice we get, 


$$
\begin{aligned}
W^{\prime \prime} & =\left(c_{\phi} c_{\alpha}^{\prime}-c_{\alpha} c_{\phi}^{\prime}\right)^{\prime \prime} \\
& =c_{\phi} c_{\alpha}^{\prime \prime \prime}-c_{\alpha} c_{\phi}^{\prime \prime \prime}+c_{\phi}^{\prime} c_{\alpha}^{\prime \prime}-c_{\phi}^{\prime \prime} c_{\alpha}^{\prime} .
\end{aligned}
$$

Using Lemma 1.5(a), (b) we may convert the third derivatives to first derivatives. We get,

$$
W^{\prime \prime}=-\left(\alpha^{2}+R(s)\right) W+c_{\phi}+c_{\phi}^{\prime} c_{\alpha}^{\prime \prime}-c_{\phi}^{\prime \prime} c_{\alpha}^{\prime} .
$$

Now set,

$$
H=c_{\phi}^{\prime} c_{\alpha}^{\prime \prime}-c_{\phi}^{\prime \prime} c_{\alpha}^{\prime}
$$

Now,

$$
H^{\prime}=c_{\phi}^{\prime} c_{\alpha}^{\prime \prime \prime}-c_{\phi}^{\prime \prime \prime} c_{\alpha}^{\prime} .
$$

Again using Lemma 1.5 on the third derivatives we get,

$$
H^{\prime}=c_{\phi}^{\prime}
$$

Thus $H=c_{\phi}$ because, $H(0)=0$ and $c_{\phi}(0)=0$ since $Y_{\phi}(0)=Y_{\alpha}(0)=0$. Inserting this into (1.10) we have,

$$
W^{\prime \prime}(s)+\left(\alpha^{2}+R(s)\right) W(s)=2 c_{\phi} .
$$

The initial conditions on $W$ follow from the definition of $W$, the expression for $W^{\prime}$ and the initial conditions on $c_{\phi}, c_{\alpha}$ in Lemma 1.5.

\section{The constant curvature comparison spaces}

We now will solve the ODE's in Lemma 1.5, 1.6 for the constant curvature spaces, $R=-1,0,1$. They will provide for us the requisite comparison functions in the next section. Compact pseudo-hermitian manifolds of constant negative curvature may be constructed by considering the unit co-sphere bundle over any compact Riemann surface of genus $g>1$. This co-sphere bundle can be endowed with a contact structure with constant negative curvature. See [4]. We now discuss the additional normalization needed to solve the ODE's in Lemma 1.5.

We need to attach an additional initial condition to the ODE's in Lemma 1.5 since they are of third order. The initial conditions are to be viewed as a normalization of the Jacobi fields. It is clear that these conditions have to be on the second derivatives. There are only two possible choices and obviously we demand,

$$
c_{\phi}^{\prime \prime}(0)=1, c_{\alpha}^{\prime \prime}(0)=0 .
$$

Under the initial conditions of Lemma 1.5 and (2.1) we have by a straightforward computation, 


\section{Lemma 2.1.}

$$
c_{\phi}(s)=\left\{\begin{array}{l}
(1-\cos \alpha s) / \alpha^{2}, R=0 \\
\left(1-\cos \left(\left(1+\alpha^{2}\right)^{1 / 2} s\right)\right) /\left(1+\alpha^{2}\right), \quad R=1 .
\end{array}\right.
$$

When $R=-1$ we have,

$$
c_{\phi}(s)=\left\{\begin{array}{l}
\left(\cosh \left(\left(1-\alpha^{2}\right)^{1 / 2} s\right)-1\right) /\left(1-\alpha^{2}\right), \alpha<1 \\
s^{2} / 2, \alpha=1 \\
\left(1-\cos \left(\left(\alpha^{2}-1\right)^{1 / 2} s\right)\right) /\left(\alpha^{2}-1\right), \alpha>1 .
\end{array}\right.
$$

For $c_{\alpha}$ we have the following expressions,

$$
c_{\alpha}(s)=\left\{\begin{array}{l}
(\alpha s-\sin \alpha s) / \alpha^{3}, R=0 \\
\left(\left(1+\alpha^{2}\right)^{1 / 2} s-\sin \left(\left(1+\alpha^{2}\right)^{1 / 2} s\right)\right) /\left(1+\alpha^{2}\right)^{3 / 2}, \quad R=1 .
\end{array}\right.
$$

When $R=-1$ we have,

$$
c_{\alpha}(s)=\left\{\begin{array}{l}
\left(\sinh \left(\left(1-\alpha^{2}\right)^{1 / 2} s\right)-\left(1-\alpha^{2}\right)^{1 / 2} s\right) /\left(1-\alpha^{2}\right)^{3 / 2}, \alpha<1 \\
s^{3} / 6, \alpha=1 \\
\left(\left(\alpha^{2}-1\right)^{1 / 2} s-\sin \left(\left(\alpha^{2}-1\right)^{1 / 2} s\right)\right) /\left(\alpha^{2}-1\right)^{3 / 2}, \alpha>1
\end{array}\right.
$$

We introduce the notation,

$$
\beta=\left(1+\alpha^{2}\right)^{1 / 2}, \gamma=\left(1-\alpha^{2}\right)^{1 / 2}, \sigma=\left(\alpha^{2}-1\right)^{1 / 2} .
$$

For the Wronskian we have,

$$
W(s, \alpha, \phi)=\left\{\begin{array}{l}
(2-2 \cos \alpha s-\alpha s \sin \alpha s) / \alpha^{4}, \quad R=0 \\
(2-2 \cos \beta s-\beta s \sin \beta s) / \beta^{4}, \quad R=1
\end{array}\right.
$$

When $R=-1$ we have,

$$
W(s, \alpha, \phi)=\left\{\begin{array}{l}
(2+\gamma s \sinh \gamma s-2 \cosh \gamma s) / \gamma^{4}, \alpha<1 \\
s^{4} / 12, \alpha=1 \\
(2-2 \cos \sigma s-\sigma s \sin \sigma s) / \sigma^{4}, \alpha>1 .
\end{array}\right.
$$

\section{Comparison theorems for $c_{\phi}$ and the Wronskian $W$}

We will now prove various comparison theorems. The comparison theorems are straightforward consequences of the standard Sturm comparison theorem and the method of variation of parameters for linear second order ODE. We set up some notation. We denote by $c_{\phi}$, hyp the $c_{\phi}$ for the case $R=-1$, and $c_{\phi \text {, sph }}$ the $c_{\phi}$ for the case $R=1$. The case $R=0$ will be denoted by $c_{\phi}$, hei. We use analogous notation for $c_{\alpha}$ and $W$ the Wronskian. We have, 
Lemma 3.1. Let $-1 \leq R \leq 1$. Then,

(a) $c_{\phi, \text { sph }}(s) \leq c_{\phi}(s), s \leq \pi /\left(1+\alpha^{2}\right)^{1 / 2}$

(b) $c_{\phi}(s) \leq c_{\phi, \text { hyp }}(s), s \leq s_{0}$.

Here $s_{0}$ denotes the first positive zero of $c_{\phi}^{\prime}(s)$. In the case $0 \leq R \leq 1$ we may replace the upper bound for $c_{\phi}$ by $c_{\phi, \text { hei }}$ in (b).

Proof. The proof follows by a straightforward use of the Sturm comparison theorem. We only show (a). From Lemma 1.5 and the normalization (2.1), the ODE for $c_{\phi}^{\prime}(s)$ is

$$
h^{\prime \prime}+\left(\alpha^{2}+R(s)\right) h=0, h=c_{\phi}^{\prime}(s), h(0)=0, h^{\prime}(0)=1 .
$$

Thus by Sturm comparison, we have,

$$
c_{\phi, \text { sph }}^{\prime}(s) \leq c_{\phi}^{\prime}(s) \leq c_{\phi, \text { hyp }}^{\prime}(s) .
$$

The above holds for the left inequality provided $s<\pi /\left(1+\alpha^{2}\right)^{1 / 2}$ and for the right inequality provided $s \leq s_{0}$. Since $c_{\phi \text {, sph }}(0)=c_{\phi \text {,hyp }}(0)=c_{\phi}(0)=0$, we easily get (a) by integrating the above inequality. In the case that $0 \leq R \leq 1$, we may replace the upper bound above by $c_{\phi \text {, hei }}$.

We now obtain bounds on the Wronskian function. We shall proceed by combining the method of variation of parameters with the Sturm comparison theorem.

Let $\psi_{1}(s), \psi_{2}(s)$ denote the basic solutions for,

$$
U^{\prime \prime}+\left(\alpha^{2}+R(s)\right) U=0,
$$

with the normalizations, $\psi_{1}(0)=1, \psi_{1}^{\prime}(0)=0$ and $\psi_{2}(0)=0, \psi_{2}^{\prime}(0)=1$. Now the variation of parameters method applied to the ODE for $W$ from Lemma 1.5 gives the solution

$$
W(s)=c_{1} \psi_{1}(s)+c_{2} \psi_{2}(s)-2 \psi_{1}(s) \int_{0}^{s} c_{\phi}(t) \psi_{2}(t) d t+2 \psi_{2}(s) \int_{0}^{s} c_{\phi}(t) \psi_{1}(t) d t .
$$

Next notice since $W(0)=0$ we must choose $c_{1}=0$. Since $c_{\phi}(0)=0$, and $W^{\prime}(0)=0$, we must also choose $c_{2}=0$. Thus,

$$
W(s)=-2 \psi_{1}(s) \int_{0}^{s} c_{\phi}(t) \psi_{2}(t) d t+2 \psi_{2}(s) \int_{0}^{s} c_{\phi}(t) \psi_{1}(t) d t .
$$

Now Sturm comparison yields,

$$
\cos \left(\left(1+\alpha^{2}\right)^{1 / 2} s\right) \leq \psi_{1}(s) \leq \begin{cases}\cosh \left(\left(1-\alpha^{2}\right)^{1 / 2} s\right), & \alpha<1 \\ 1, \alpha=1 & \\ \cos \left(\left(\alpha^{2}-1\right)^{1 / 2} s\right), & \alpha>1 .\end{cases}
$$


The left inequality holds for $s<\pi / 2\left(1+\alpha^{2}\right)^{1 / 2}$ and the right inequality for $s<s_{0}$, where $s_{0}$ is the first positive zero of $\psi_{1}(s)$.

Similarly we have, using the notation (2.2),

$$
\sin \beta s / \beta \leq \psi_{2}(s) \leq \begin{cases}\sinh \gamma s / \gamma, & \alpha<1 \\ s, \alpha=1 & \\ \sin \sigma s / \sigma, & \alpha>1\end{cases}
$$

Here the left inequality holds for $s<\pi /\left(1+\alpha^{2}\right)^{1 / 2}$, and the right inequality for $s<s_{1}$ where $s_{1}$ is the first positive zero of the function $\psi_{2}(s)$. We now substitute the bounds for $c_{\phi}$ from Lemma 3.1 and the upper and lower bounds for $\psi_{1}(s)$ and $\psi_{2}(s)$ into (3.1) and derive bounds for our Wronskian function. A straightforward computation yields:

Lemma 3.2. Let $s<\pi / 2\left(1+\alpha^{2}\right)^{1 / 2}$. Then using the notation (2.2), for $-1 \leq$ $R \leq 1$,

$$
\begin{aligned}
W(s) \geq & \left(4 \sin ^{2} \beta s-\sin \beta s \sin 2 \beta s-2 \beta s \sin \beta s\right) / 2 \beta^{4} \\
& + \begin{cases}\left(4 \cos ^{2} \sigma s-\cos \sigma s \cos 2 \sigma s-3 \cos \sigma s\right) / 2 \sigma^{4}, & \alpha>1 \\
-s^{4} / 4, & \alpha=1 \\
\left(4 \cosh ^{2} \gamma s-\cosh \gamma s \cosh 2 \gamma s-3 \cosh \gamma s\right) / 2 \gamma^{4}, & \alpha<1 .\end{cases}
\end{aligned}
$$

A similar computation now gives us the upper bounds:

Lemma 3.3. Using the notation (2.2), $-1 \leq R \leq 1$,

$$
\begin{aligned}
W(s) \leq & \left(4 \cos ^{2} \beta s-\cos \beta s \cos 2 \beta s-3 \cos \beta s\right) / 2 \beta^{4} \\
& + \begin{cases}\left(4 \sin ^{2} \sigma s-\sin \sigma s \sin 2 \sigma s-2 \sigma s \sin \sigma s\right) / 2 \sigma^{4}, & \alpha>1 \\
s^{4} / 3, & \alpha=1 \\
\left(2 \gamma s \sinh \gamma s+\sinh \gamma s \sinh 2 \gamma s-4 \sinh ^{2} \gamma s\right) / 2 \gamma^{4}, & \alpha<1 .\end{cases}
\end{aligned}
$$

In both lemmas above if $0 \leq R \leq 1$, we may replace the expressions involving hyperbolic functions with the Wronskian expression for $R=0$ in Lemma 2.1.

\section{Another Wronskian}

In the following, we consider the Wronskian associated to the volume density of the exponential map associated to a closed geodesic $\gamma(s) ; 0 \leq s \leq l$ : we shoot out unit speed contact geodesic from $\gamma(s)$ with initial direction normal to $\gamma^{\prime}(s)$. In 
this way we parametrize a tubular neighborhood of the geodesic $\gamma$, and we wish to determine the ODE for the volume density of this exponential map.

Consider the Jacobi field,

$$
Y_{s}(t)=c_{s}(t) \alpha X+c_{s}^{\prime}(t) J X+c_{s}(t) T .
$$

The differential equation satisfied by $c_{S}(t)$ is according to Lemma 1.5:

$$
\left(c_{s}^{\prime \prime}+\alpha^{2} c_{s}\right)^{\prime}+R(s) c_{s}^{\prime}=0 .
$$

Thus to solve this ODE we need to supplement it by three initial conditions. In the situation we are faced with we are looking at the focal point situation of Jacobi fields, a situation well-known in the theory of geometric optics. Thus an end-point lies on a curve $\gamma(s)$ which we are assuming to be a geodesic with curvature $\tau$. We claim that the initial conditions are:

$$
c_{s}(0)=0, c_{s}^{\prime}(0)=1, c_{s}^{\prime \prime}(0)=\tau .
$$

To check the last initial condition we will again use the fact that we are assuming the torsion vanishes.

From the curve $\gamma(s)$ we will shoot out geodesics with curvature $\alpha$. Thus we are looking at a surface,

$$
f(t, s)=\exp _{\gamma(s)}(t X)
$$

From (4.3) we note that

$$
Y_{S}(0)=\frac{\partial f}{\partial s}(0,0)=J X .
$$

Thus $c_{s}(0)=0, c_{s}^{\prime}(0)=1$.

We now check the last initial condition. Let $v=J X$ a tangent vector to our geodesic $\gamma(s)$. Since the torsion vanishes we have the following at $t=0$,

$$
\left\langle\nabla_{\frac{\partial}{\partial t}} \frac{\partial f}{\partial s}, v\right\rangle=\left\langle\nabla_{\frac{\partial}{\partial s}} \frac{\partial f}{\partial t}, v\right\rangle .
$$

This means,

$$
\left\langle Y_{s}^{\prime}, v\right\rangle=\left\langle\nabla_{J X} X, v\right\rangle
$$

Thus

$$
Y_{s}^{\prime}-\nabla_{J X} X \in T_{\gamma(s)}^{\perp}
$$

that is to say the quantity in (4.5) must have no component at $t=0$ in the direction of the tangent vector to $\gamma(s)$ thus no component involving $J X$. Now note the coefficient of the component of $Y_{s}^{\prime}(0)$ involving $J X$ is by differentiation of (4.1), (here we have used the fact that $\left.c_{s}(0)=0\right)$

$$
c_{s}^{\prime \prime}(0)
$$


Next by the geodesic equation of Rumin [6],

$$
\nabla_{J X}(X)=-J^{2} \nabla_{J X}(X)=-J \nabla_{J X}(J X)=\tau J X .
$$

Thus from (4.5), (4.6) and (4.7),

$$
Y_{s}^{\prime}(0)-\nabla_{J X} X=a X+\left(c_{s}^{\prime \prime}(0)-\tau\right) J X+c T .
$$

By (4.5) we must therefore have $c_{s}^{\prime \prime}(0)=\tau$.

We now have three vector fields, $X, Y_{s}, Y_{\alpha}$, where $Y_{\alpha}$ is as before and the coefficient $c_{\alpha}(t)$ satisfies the ODE of Lemma 1.5(b) with initial conditions of Lemma 1.5(b) and that given by (2.1). The coefficients of the Jacobi field $Y_{S}(t)$ satisfies (1a) above. Let

$$
\mathcal{W}(t)=\Theta \wedge d \Theta\left(X, Y_{s}, Y_{\alpha}\right)
$$

Then we note,

$$
\mathcal{W}(t)=c_{s} c_{\alpha}^{\prime}-c_{\alpha} c_{s}^{\prime}
$$

exactly as in Lemma 1.6. The attendant ODE for $\mathcal{W}(t)$ follows from Lemma 1.6, and the initial conditions for $\mathcal{W}(t)$ are the same as in Lemma 6 , because, $c_{s}(0)=$ $c_{\alpha}(0)=0$. Thus we have,

$$
\mathcal{W}^{\prime \prime}+\left(\alpha^{2}+R(t)\right) \mathcal{W}=2 c_{s}(t), \mathcal{W}(0)=0, \mathcal{W}^{\prime}(0)=0
$$

We now wish to solve (4.9). To do so we first solve for $c_{S}(t)$. In (4.2) we set $c_{s}^{\prime}(t)=U(t)$ as before, and so consider,

$$
U^{\prime \prime}(t)+\left(\alpha^{2}+R(s)\right) U=0, U(0)=1, U^{\prime}(0)=\tau .
$$

A straightforward application of the Sturm comparison theorem as in Lemma 3.1, yields with $\beta=\left(1+\alpha^{2}\right)^{1 / 2}, \sigma=\left(\alpha^{2}-1\right)^{1 / 2}, \gamma=\left(1-\alpha^{2}\right)^{1 / 2}$.

$$
\cos \beta t+\tau \frac{\sin \beta t}{\beta} \leq U(t), t \leq t_{0}
$$

where $t_{0}$ is the first zero of the left side of (4.10). Note $t_{0} \geq \pi / 2 \beta$. Likewise Sturm comparison yields,

$$
U(t) \leq \begin{cases}\cos \sigma t+\tau \frac{\sin \sigma t}{\sigma}, & |\alpha|>1, \\ 1+\tau t, \alpha= \pm 1, & \\ \cosh \gamma t+\tau \frac{\sinh \gamma t}{\gamma}, & |\alpha|<1 .\end{cases}
$$

Integrating $U(t)$ and using $c_{s}(0)=0$ we get,

$$
\frac{\sin \beta t}{\beta}+\frac{\tau}{\beta^{2}}(1-\cos \beta t) \leq c_{S}(t), t \leq t_{0}
$$


and

$$
c_{s}(t) \leq \begin{cases}\frac{\sin \sigma t}{\sigma}+\frac{\tau}{\sigma^{2}}(1-\cos \sigma t), & |\alpha|>1 \\ t+\frac{\tau}{2} t^{2}, & \alpha= \pm 1 \\ \frac{\sinh \gamma t}{\gamma}+\frac{\tau}{\gamma^{2}}(\cosh \gamma t-1), & |\alpha|<1 .\end{cases}
$$

The first line of (4.12) holds to the first zero $t_{1}$ of $U(t)$ and as we have remarked above we note $t_{1} \geq \pi / 2 \beta$. Thus for large $|\alpha|$ we have $t_{1} \sim \pi / 2 \alpha$.

Now as before we will use (4.12) with our variation by parameters formula (3.1). The functions $\psi_{1}(t), \psi_{2}(t)$ and the bounds for them below (3.1) will remain the same. So,

$$
\mathcal{W}(t)=-2 \psi_{1}(t) \int_{0}^{t} c_{s}(x) \psi_{2}(x) d x+2 \psi_{2}(t) \int_{0}^{t} c_{s}(x) \psi_{1}(x) d x .
$$

We need upper bounds for the second integral and lower bounds for the first integral. Now,

$$
\psi_{1}(t) \geq \cos \beta t, \psi_{2}(t) \geq \frac{\sin \beta t}{\beta} .
$$

Thus,

$$
-2 \psi_{1}(t) \int_{0}^{t} c_{s}(x) \psi_{2}(x) d x \leq-2 \cos \beta t \int_{0}^{t}\left(\frac{\sin \beta x}{\beta}+\frac{\tau}{\beta^{2}}(1-\cos \beta x)\right) \frac{\sin \beta x}{\beta} d x .
$$

An easy computation shows that the integral on the right above is,

$$
\frac{\tau}{2 \beta^{4}}\left(4 \cos ^{2} \beta t-\cos \beta t \cos 2 \beta t-3 \cos \beta t\right)+\frac{\cos \beta t}{2 \beta^{3}}(\sin 2 \beta t-2 \beta t) .
$$

Next we find upper bounds for

$$
2 \psi_{2}(t) \int_{0}^{t} c_{s}(x) \psi_{(x)} d x
$$

There are three cases to consider.

Case 1. $|\alpha|>1$.

$$
2 \psi_{2}(t) \int_{0}^{t} c_{s}(x) \psi(x) d x \leq 2 \frac{\sin \sigma t}{\sigma} \int_{0}^{t}\left(\frac{\sin \sigma x}{\sigma}+\frac{\tau}{\sigma^{2}}(1-\cos \sigma x)\right) \cos \sigma x d x .
$$

Computing the integral above we have,

$$
\begin{aligned}
2 \psi_{2}(t) \int_{0}^{t} c_{s}(x) \psi(x) d x \leq & \frac{\tau}{2 \sigma^{4}}\left(4 \sin ^{2} \sigma t-\sin \sigma t \sin 2 \sigma t-2 \sigma t \sin \sigma t\right) \\
& +\frac{\sin \sigma t}{2 \sigma^{3}}(1-\cos 2 \sigma t) .
\end{aligned}
$$


Case 2. $\alpha= \pm 1$.

$$
2 \psi_{2}(t) \int_{0}^{t} c_{s}(x) \psi(x) d x \leq 2 t \int_{0}^{t}\left(x+\frac{\tau}{2} x^{2}\right) d x=t^{3}\left(1+\frac{1}{3} \tau t\right) .
$$

Case 3. $|\alpha|<1$.

$$
2 \psi_{2}(t) \int_{0}^{t} c_{s}(x) \psi(x) d x \leq 2 \frac{\sinh \gamma t}{\gamma} \int_{0}^{t}\left(\frac{\sinh \gamma x}{\gamma}+\frac{\tau}{\gamma^{2}}(\cosh \gamma x-1)\right) \cosh \gamma x d x .
$$

Thus,

$$
\begin{aligned}
2 \psi_{2}(t) \int_{0}^{t} c_{s}(x) \psi(x) d x \leq & \frac{\tau}{2 \gamma^{4}}\left(2 \gamma t \sinh \gamma t+\sinh \gamma t \sinh 2 \gamma t-4 \sinh ^{2} \gamma t\right) \\
& +\frac{\sinh \gamma t}{2 \gamma^{3}}(\cosh 2 \gamma t-1) .
\end{aligned}
$$

Putting the estimates (4.15)-(4.17) together we get,

$$
\begin{aligned}
\mathcal{W}(t, \alpha, s) \leq & \frac{\tau}{2 \beta^{4}}\left(4 \cos ^{2} \beta t-\cos \beta t \cos 2 \beta t-3 \cos \beta t\right) \\
& +\frac{\cos \beta t}{2 \beta^{3}}(\sin 2 \beta t-2 \beta t) \\
& + \begin{cases}\frac{\tau}{2 \sigma^{4}}\left(4 \sin ^{2} \sigma t-\sin \sigma t \sin 2 \sigma t-2 \sigma t \sin \sigma t\right) & \sin \sigma t \\
+\frac{2}{2 \sigma^{3}}(1-\cos 2 \sigma t), & |\alpha|>1 \\
t^{3}\left(1+\frac{1}{3} \tau t\right), & \alpha= \pm 1 \\
\frac{\tau}{2 \gamma^{4}}\left(2 \gamma t \sinh \gamma t+\sinh \gamma t \sinh 2 \gamma t-4 \sinh ^{2} \gamma t\right) \\
+\frac{\sinh \gamma t}{2 \gamma^{3}}(\cosh 2 \gamma t-1), & |\alpha|<1 .\end{cases}
\end{aligned}
$$

\section{Geodesic flow and volume preservation}

We shall reason under the assumption of zero Webster torsion as before (1.1). We develop some notation. We have the frame vectors $\left\{e_{1}, e_{2}, T\right\}$. By a co-vector we mean

$$
\xi=\xi_{1} d x_{1}+\xi_{2} d x_{2}+\xi_{3} \Theta .
$$

The symbol of $e_{i}$ is $\left\langle e_{i}, \xi\right\rangle$, where $\langle$,$\rangle is the standard pairing between tangent and$ co-tangent vectors. We have: 
Lemma 5.1. Consider the Hamiltonian,

$$
H(x, \xi)=\frac{1}{2}\left(\left\langle e_{1}, \xi\right\rangle^{2}+\left\langle e_{2}, \xi\right\rangle^{2}\right) .
$$

Then the integral curves of the Hamilton-Jacobi equations for H project to geodesics in the base projection.

Moreover, along the integral curves in the phase space, $\xi_{3}(s)=\alpha / 2$, for some constant $\alpha$.

Furthermore, $H \neq 0$ along the integral curves of the Hamilton Jacobi equation That is there are no abnormal geodesics.

Proof. We reason at a fixed point $P$ in the base. Since we are at a fixed point, we may assume that at this point, the connection tensor vanishes and moreover at $P$ we can arrange,

$$
\left\langle e_{i}, d x_{j}\right\rangle=\delta_{i j}, 1 \leq i, j \leq 2 .
$$

We also have by $[2,($ Appendix) $]$ that at $P$,

$$
\left[\frac{\partial}{\partial x_{i}}, e_{j}\right]= \begin{cases}0, & i=j \\ -2 T, & i=1, j=2\end{cases}
$$

Now the Hamilton-Jacobi equations are,

$$
\begin{aligned}
& x_{1}^{\prime}(s)=\left\langle e_{1}, \xi\right\rangle\left\langle e_{1}, d x_{1}\right\rangle+\left\langle e_{2}, \xi\right\rangle\left\langle e_{2}, d x_{1}\right\rangle=w_{1} \\
& x_{2}^{\prime}(s)=\left\langle e_{1}, \xi\right\rangle\left\langle e_{1}, d x_{2}\right\rangle+\left\langle e_{2}, \xi\right\rangle\left\langle e_{2}, d x_{2}\right\rangle=w_{2} \\
& x_{3}^{\prime}(s)=0, \text { since } \Theta\left(e_{i}\right)=0 .
\end{aligned}
$$

Clearly (5.5) tells us that the base projection is already Legendrian since the tangent vector to the base projection curve is

$$
X=w_{1} e_{1}+w_{2} e_{2}
$$

Now the rest of the Hamilton-Jacobi equations are,

$$
\xi_{1}^{\prime}(s)=-\left\langle e_{1}, \xi\right\rangle\left\langle\left[\frac{\partial}{\partial x_{1}}, e_{1}\right], \xi\right\rangle-\left\langle e_{2}, \xi\right\rangle\left\langle\left[\frac{\partial}{\partial x_{1}}, e_{2}\right], \xi\right\rangle .
$$

At $P$ the right side by (5.2) is,

$$
2 \xi_{3}\left\langle e_{2}, \xi\right\rangle
$$

Similarly,

$$
\xi_{2}^{\prime}(s)=-2 \xi_{3}\left\langle e_{1}, \xi\right\rangle
$$

Lastly,

$$
\xi_{3}^{\prime}(s)=-\left\langle e_{1}, \xi\right\rangle\left\langle\left[T, e_{1}\right], \xi\right\rangle-\left\langle e_{2}, \xi\right\rangle\left\langle\left[T, e_{2}\right], \xi\right\rangle .
$$


The righthand side of the last identity is given by

$$
-\left\langle e_{1}, \xi\right\rangle\left\langle e_{2}, \xi\right\rangle \omega(T)+\left\langle e_{2}, \xi\right\rangle\left\langle e_{1}, \xi\right\rangle \omega(T)=0
$$

on account of the formulae:

$$
\begin{aligned}
& {\left[e_{1}, T\right]=\left(\Re A_{11}\right) e_{1}-\left(\left(\Im A_{11}\right)+\omega(T)\right) e_{2}} \\
& {\left[e_{2}, T\right]=\left(\left(\Im A_{11}\right)+\omega(T)\right) e_{1}+\left(\Re A_{11}\right) e_{2} .}
\end{aligned}
$$

We remark that this conclusion is independent of the framing chosen.

We set,

$$
\xi_{3}(s)=\alpha / 2 .
$$

Now we compute, $\nabla_{X} X$. From (5.6) and since the computation is being done at a point, we may assume the Tanaka connection vanishes at that point, and so

$$
\nabla_{X} X=w_{1}^{\prime}(s) e_{1}+w_{2}^{\prime}(s) e_{2}
$$

From (5.3), (5.4), again using the connection vanishes at $P$, and (5.1),

$$
w_{1}^{\prime}(s)=\left\langle e_{1}, \xi^{\prime}\right\rangle=\alpha \xi_{2} .
$$

Where we used (5.7) and (5.9) to obtain the last equality. Using (5.8) and (5.9) we get,

$$
w_{2}^{\prime}(s)=-\alpha \xi_{1}
$$

Substituting the last two identities into (5.10) we get,

$$
\nabla_{X} X=\alpha \xi_{2} e_{1}-\alpha \xi_{1} e_{2}
$$

At $P$ we also have,

$$
w_{1}=\xi_{1}, w_{2}=\xi_{2},
$$

thus at $P$,

$$
X=\xi_{1} e_{1}+\xi_{2} e_{2} .
$$

Using $J\left(e_{1}\right)=-e_{2}, J\left(e_{2}\right)=e_{1}$ we see,

$$
\nabla_{X} X=\alpha J X
$$

That is the base projection is a geodesic by Lemma 1.1 .

To show there are no abnormal geodesics, we first observe that the set $H=0$, is a symplectic manifold with respect to the fundamental symplectic form $\lambda$ of the cotangent bundle $T^{\star} M$ of the manifold $M$. To see this, note $H=0$ is given by the vanishing of the two symbols $\left\langle e_{1}, \xi\right\rangle=\sigma_{1}(x, \xi)$ and $\left\langle e_{2}, \xi\right\rangle=\sigma_{2}(x, \xi)$. By [2] and (5.2), the Poisson bracket,

$$
\left\{\sigma_{1}, \sigma_{2}\right\}=\left\langle\left[e_{1}, e_{2}\right], \xi\right\rangle=\langle-2 T, \xi\rangle=-2 \xi_{3} \neq 0 .
$$


The first identity in (5.11) is standard for vector fields, see Treves [8, page 39, Corollary 4.2]. The last inequality in (5.11), follows from the claim $\xi_{3} \neq 0$ on $H=$ 0 . For if $\xi_{3}=0$, on $H=0$, by (5.1), $\xi_{1}=\xi_{2}=0$ and we fall into the zero section of the cotangent bundle which is excluded from the characteristic set. Thus the characteristic set is defined by the vanishing of two functions whose Poisson bracket is a non-vanishing function. Thus the characteristic set is symplectic. Now if there is an integral curve $\gamma(t)$ of the Hamilton Jacobi eqn lying on the characteristic set, we have, for every tangent vector $v$ to the sub-manifold $H=0$,

$$
\lambda\left(\gamma^{\prime}(t), v\right)=d H(v)=0 .
$$

That is if we denote $H=0$ by $\Sigma$, we have just checked, $\gamma^{\prime}(t) \in T \Sigma \cap T \Sigma^{\perp}$ where $\perp$ is understood in the symplectic sense, $\lambda(v, w)=0$. But $\Sigma$ is symplectic, and so $T \Sigma \cap T \Sigma^{\perp}=\{0\}$. We have a contradiction. Hence there are no abnormal geodesics.

\section{The $A$-injectivity radius}

In order to develop some control of the geometry we formulate the concept of the $A$ injectivity radius. Under the assumption of vanishing torsion, the curvature $\alpha$ of a geodesic is constant, and hence may be considered as a parameter. For each $p \in M$ and real number $\alpha$ let

$$
l(p, \alpha)=\sup \left\{\tau \mid \gamma_{\xi, \alpha}(t) \text { is minimizing for each } \xi, 0<t<\tau\right\} .
$$

Let us define the $A$ injectivity radius $i_{A}(p)$ at a point $p \in M$ :

$$
i_{A}(p)=\sup \left\{\tau \mid \gamma_{\xi, \alpha}(t) \text { is minimizing for all }|\alpha|<A, 0<t<\tau\right\} .
$$

Thus we say a point $q$ is in the $A$ cut-locus if $q=\gamma_{\xi, \alpha}(l)$ for some $\xi$ and some $\alpha \leq A$ and that it is the first point along this $\alpha$ geodesic beyond which the geodesic no longer minimizes distance to $p$.

For each point $p \in M$, we wish to bound from below the region in the $\alpha, l$ plane determined by the function $l(p, \alpha)$. There are two possible situations according to whether $l$ is a monotone decreasing function of $|\alpha|$. In either case, we note the asymptotic behavior from Lemma 3.2 and Lemma 3.3:

Lemma 6.1. For large values of $\alpha$ we have

$$
l(p, \alpha) \alpha \sim \pi / 2
$$

In the more complicated case where $l$ is not a monotone function of $\alpha$, we show that each local minimum of $l$ satisfies a uniform lower bound. Let us denote $\operatorname{Vol}(M)=$ $V(M)$, and $d(M)$ to be the diameter of $M$. 
Proposition 6.2. Assume $V(M) \geq V_{0}, d(M) \leq d_{0}$. Suppose $l_{0}=l\left(p, \alpha_{0}\right)$ is a local minimum, and $l(p, A)>l_{0}$ for some $A>\alpha_{0}$. Then

$$
l_{0} \geq C=C\left(V_{0}, d_{0}, A\right) .
$$

Proof. It follows from the assumption that there is a point $q=\gamma_{\xi_{0}, \alpha_{0}}\left(l_{0}\right)$ which realizes the minimal distance from $p$ to its $A$ cut-locus, and we may assume without loss of generality that this is not a conjugate point. We claim that there is at least another $\alpha^{\prime}$ geodesic (with $\alpha^{\prime} \leq A$ ) issuing from $p$ of length $l_{0}$ ending at $q$ : There is a sequence $l_{i}>l_{0}$ converging to $l_{0}$, a sequence of unit contact tangent vectors $\xi_{i}$ at $p$, a sequence $\alpha_{i} \leq A$ and a sequence of points $q_{i}=\gamma_{\xi_{0}, \alpha_{0}}\left(l_{i}\right)=\gamma_{\xi_{i}, \alpha_{i}}\left(l_{i}^{\prime}\right)$ where $l_{i}^{\prime} \leq l_{i}$. By compactness, a subsequence $\xi_{i}$ converges to $\xi^{\prime}, \alpha_{i}^{\prime}$ converges to $\alpha^{\prime}$ and the corresponding geodesics converges to the required $\alpha^{\prime}$ geodesic. points

We claim $\gamma_{\xi_{0}, \alpha_{0}}^{\prime}\left(l_{0}\right)=-\gamma_{\xi^{\prime}, \alpha^{\prime}}^{\prime}\left(l_{0}\right)$ : For if not, then the surfaces formed by the

$$
\left\{\gamma_{\xi, \alpha}\left(l_{0}\right) \mid \text { for } \xi \text { close to } \xi_{0}, \alpha \text { close to } \alpha_{0}\right\}
$$

and that formed by the points

$$
\left\{\gamma_{\xi, \alpha}\left(l_{0}\right) \text { for } \xi \text { close to } \xi^{\prime}, \alpha \text { close to } \alpha^{\prime}\right\}
$$

will meet transversly at $q$. Hence for $\epsilon$ sufficiently small, the surfaces formed by the points

$$
\left\{\gamma_{\xi, \alpha}\left(l_{0}-\epsilon\right) \text { for } \xi \text { close to } \xi_{0}, \alpha \text { close to } \alpha_{0}\right\}
$$

and that formed by the points

$$
\left\{\gamma_{\xi, \alpha}\left(l_{0}-\epsilon\right) \text { for } \xi \text { close to } \xi^{\prime}, \alpha \text { close to } \alpha^{\prime}\right\}
$$

will intersect at a point which will be in the $A$ cut-locus of $p$ but closer than $q$. This contradicts the choice of $q$. We can then reverse the role played by $p$ and $q$ in the argument above to show that the two geodesics from $p$ to $q$ must piece up to form a closed $C^{1}$ contact curve $\Gamma$ of length $2 l_{0}$. Now we evaluate the volume of $M$ by considering the volume of geodesic tubes around this $\Gamma$ : For each point $\Gamma(s)$ let $\xi(s)$ be the unit contact vector orthogonal to $\Gamma^{\prime}(s)$, and $\gamma_{\xi(s), \alpha}(t)$ be the unit speed $\alpha$ geodesic issuing from $\Gamma(s)$ in the direction $\xi(s)$; such a geodesic minimizes distance to $\Gamma(s)$ for $-t(\xi(s), \alpha)<t<T(\xi(s), \alpha)$. It follows from the argument of Gromov, Bellaiche [1] that any point $q \in M$ may be joined to $\Gamma$ via one of these geodesics. Thus we may compute the volume of $M$ via Fubini's theorem:

Recall,

$$
V(M) \geq V_{0}, d(M) \leq d_{0} .
$$

We also assume that the curve $\gamma(s)$ is a closed geodesic loop of total length $l_{0}$. Notice from (4.18) that the upper bounds for $\mathcal{W}(t, \alpha, s)$ are independent of $s$. Further in the $\alpha, t$ plane since we are interested in upper bounds we may always integrate $\mathcal{W}(t, \alpha, s)$ upto the conjugate locus. In the $\alpha, t$ plane consider the region $R$,

$$
R=\left\{(t, \alpha)\left|t \leq d_{0},\right| \alpha \mid \leq 2\right\} \cup\left\{(t, \alpha)\left|0 \leq t \leq \frac{10}{|\alpha|},\right| \alpha \mid>2\right\}=R_{1} \cup R_{2} .
$$


Thus from (6.1),

$$
V_{0} \leq \int_{0}^{l_{0}} \int_{R} \mathcal{W}(t, \alpha, s) d t d \alpha d s
$$

By the estimates (4.18),

$$
\int_{0}^{l_{0}} \int_{R} \mathcal{W}(t, \alpha, s) d t d \alpha d s \leq l_{0}\left(c \int_{R_{1}}(1+\tau) e^{3 d_{0}} d \alpha d t+\int_{R_{2}} t^{3}\left(1+\frac{\tau}{3} t\right) d t d \alpha\right) .
$$

The expression to the right is bounded by,

$$
l_{0}\left(c d_{0}(1+\tau) e^{3 d_{0}}+\int_{|\alpha|>2} \int_{0}^{\frac{10}{\alpha}} t^{3}\left(1+\frac{\tau}{3} t\right) d t d \alpha\right)
$$

In the expression above $c$ is independent of $\tau, d_{0}, l_{0}$ and $V_{0}$. Thus we get,

$$
V_{0} \leq c_{0} l_{0}(1+\tau)\left(1+d_{0} e^{3 d_{0}}\right) .
$$

Again $c_{0}$ is independent of $l_{0}, d_{0}, V_{0}$ and $\tau$. Thus from (6.2) it follows that $l_{0}$ is bounded below under the assumptions (6.1). Therefore, we find a lower bound for $l_{0}$ depending only on $\operatorname{Vol}(M)$ and the diameter $d(M)$.

\section{The Santalo formula and the isoperimetric inequality}

We now wish to prove a version of the isoperimetric inequality on CR manifolds. We begin with a version of the Santalo formula.

On our base CR manifold $M$, we have a global contact form $\Theta$, and a global volume form $d V=\Theta \wedge d \Theta$. We first have the unit contact bundle over $M$ that we will denote by $S_{c} M$, and $\pi: S_{c} M \rightarrow M$ the projection to the base. There is a natural Liouville measure $d \mu$ on $S_{c} M$, given by,

$$
d \mu=\Theta \wedge d \Theta \wedge d \phi .
$$

Lemma 7.1. Let us denote the Hamiltonian vector field by $W$, then

$$
\mathcal{L}_{W}(d \mu)=0,
$$

where $\mathcal{L}_{W}$ denote the Lie derivative.

Proof. It is well-known that

$$
\mathcal{L}_{W}(d \mu)=\operatorname{div}_{\Lambda_{\alpha}}(W) d \mu .
$$

Since the $W$ is tangent to $\Lambda_{\alpha}$, it follows that

$$
\mathcal{L}_{W}(d \mu)=\operatorname{div} W d \mu .
$$

The latter vanishes since $W$ arises from a Hamiltonian. 
Thus the Liouville measure $d \mu$ is also preserved. Let $\Psi_{t}$ denote the Hamiltonian flow given by Lemma 7.1. This flow preserves the Liouville measure $d \mu$. Furthermore by Lemma 1.2, $\alpha$ is preserved along the flow. Thus we have

$$
\int_{S_{c} M} f(\phi, \alpha) d \mu=\int_{M} \Theta \wedge d \Theta \int_{S^{1}} f(\phi, \alpha) d \phi .
$$

In addition, the zero torsion assumption shows that each geodesic has constant curvature $\alpha$ thus we may regard $\alpha$ as a parameter. Thus for each value of $\alpha$ the unit contact bundle is foliated by the set of $\alpha$ geodesics $\Lambda_{\alpha}$ (the geodesics with curvature equal to $\alpha$ ). It will be convenient to view this foliation as a foliation of $S_{c} M \times R$ so that each copy $S_{c} M \times\{\alpha\}$ is identified with $\Lambda_{\alpha}$. Let $\gamma_{\xi, \alpha}(t)$ denote the unit speed geodesic with initial velocity $\xi$ and curvature $\alpha$, then the geodesic flow on $\Lambda_{\alpha}$ is given by $\Psi_{t}(\xi, \alpha)=\left(\gamma_{\xi, \alpha}^{\prime}(t), \alpha\right)$.

Let $S_{p}$ denote the unit contact vectors over the point $p$. We then have the following analogue of Fubini's theorem:

Lemma 7.2. For each $\alpha$, we have

$$
\int_{\Lambda_{\alpha}} f(\xi) d \mu=\frac{1}{2 \pi} \int_{M}\left(\int_{S_{p}} f(\xi) d \phi(\xi)\right) \Theta \wedge d \Theta(p) .
$$

Now we are going to prove an analogue of the Santalo formula. Consider $\Omega$ a relatively compact domain in $M$ with smooth boundary.

Define for each $\xi, \alpha$ :

$$
\tau(\xi, \alpha)=\sup \left\{\tau>0, \gamma_{\xi, \alpha}(t) \in \Omega \text { for all } 0<t<\tau\right\} .
$$

That is, if $\tau(\xi, \alpha)<\infty$, then $\gamma_{\xi, \alpha}(\tau(\xi, \alpha))$ will be the first point on the geodesic $\gamma_{\xi, \alpha}(t)$ to hit $\partial \Omega$. Let $c(\xi, \alpha)$ denote the distance from the base projection $\pi(\xi)$ to its cut-point along $\gamma \xi, \alpha$.

Define,

$$
l(\xi, \alpha)=\inf \{c(\xi, \alpha), \tau(\xi, \alpha)\}
$$

and

$$
(U \Omega)_{\alpha}=\{\xi: c(\xi, \alpha) \geq \tau(\xi, \alpha)\} .
$$

Now consider the boundary $\partial \Omega$. Let $v$ denote the inward unit Legendrian normal along $\partial \Omega$. From the definition of $\Lambda_{\alpha}$, define

$$
\Lambda_{\alpha}^{+}(\partial \Omega)=\left\{\eta \in \Lambda_{\alpha} \mid \eta \cdot v>0\right\}
$$

The foliation $\Lambda_{\alpha}^{+}(\partial \Omega)$ is equipped with the measure

$$
d \sigma(\eta)=d \phi \wedge d A
$$

where $d A$ denotes the surface measure on $\partial \Omega$. We have now the analogue of Santalo's formula: 
Lemma 7.3. For all integrable functions $f$ on $\Lambda_{\alpha}$ we have:

$$
\int_{\Lambda_{\alpha}(\Omega)} f d \mu=\int_{\Lambda_{\alpha}^{+}(\partial \Omega)} \eta \cdot v \int_{0}^{\tau(\xi, \alpha)} f\left(\Psi_{t}(\eta)\right) d t \wedge d \sigma(\eta)
$$

and

$$
\int_{(U \Omega)_{\alpha}(\Omega)} f d \mu=\int_{\Lambda_{\alpha}^{+}(\partial \Omega)} \eta \cdot v \int_{0}^{l(\eta, \alpha)} f\left(\Psi_{t}(\eta)\right) d t \wedge d \sigma(\eta) .
$$

Proof. This follows from the invariance of the measure $d \mu$ under the geodesic flow:

$$
d \mu\left(\Psi_{t}(\eta)\right)=\left(\Psi_{t}\right)_{*} d \mu(\eta)=\left(\Psi_{t}\right)_{*}(\Theta \wedge d \Theta \wedge d \phi)(\eta)
$$

Hence, denoting by $s$ the distance from $\partial \Omega$, we have

$$
\begin{aligned}
d \mu\left(\Psi_{t}(\eta)\right) & =(d \Psi)_{*} d s \wedge d A \wedge d \phi \\
& =\left(\Psi_{t}\right)_{*} \frac{d s}{d t} d t \wedge d \sigma \\
& =\eta \cdot v d t d \sigma .
\end{aligned}
$$

Now we bring in the notion of visibility angle. For each point $p \in M$ let:

$$
V_{p, \alpha}=\left\{\xi \in(U \Omega)_{\alpha}, \pi(\xi)=p\right\}
$$

We then define the visibility angle

$$
\omega_{\alpha}(p)=\frac{1}{2 \pi} \int_{V_{p, \alpha}} d \phi(\xi) .
$$

Lemma 7.4. Let $\left(M^{3}, \Theta\right)$ be compact with Webster curvature satisfying $R>-c$. Let $d(M)$ denote the diameter of $M$. Let $\Sigma$ be any compact surface dividing $M$ into domains $M_{1}, M_{2}$ with $\partial M_{1}=\partial M_{2}=\Sigma$. Then if $\Omega=M_{1}$ has smaller volume than $M_{2}$, we have for all $p \in M_{1}$,

$$
\int_{-A}^{A} \omega_{\alpha}(p) d \alpha \geq C V(M)-C_{1} / A^{4}
$$

where the constants $C, C_{1}$ depends only on $d(M), \operatorname{Vol}(M)$.

Proof. We have for each point $q \in M_{2}$ there is a unit speed length minimizing geodesic starting at $p \in M_{1}$ with initial tangent vector $\xi$ of curvature $\alpha$ joining $p$ to 
$q$ and this geodesic must hit $\partial M_{1}$ say at time $t(\xi, \alpha)$ and this geodesic continues to minimize length until time $T(\xi, \alpha)$, thus we may compute the volume of $M_{2}$ :

$$
\begin{aligned}
\operatorname{Vol}\left(M_{2}\right) & =\int_{-\infty}^{\infty} \int_{V_{p, \alpha}} \int_{t(\xi, \alpha)}^{T(\xi, \alpha)} W(t, \xi(s), \alpha) d t d \xi d \alpha \\
& \leq \int_{-\infty}^{\infty} \int_{V_{p, \alpha}} \int_{0}^{d(M)} W_{0}(t, \alpha) d t d \xi d \alpha \\
& \leq C \int_{-\infty}^{\infty} \omega_{\alpha}(p) d \alpha .
\end{aligned}
$$

In the second to last line, $W_{0}(t, \alpha)$ denotes the Wronskian in the in the comparison space of Webster curvature $c$. If we cut off the $\alpha$-integration, we observe that for $\alpha$ large, $l(\eta, \alpha) \sim c / \alpha$, and hence

$$
\operatorname{Vol}\left(M_{2}\right)-C / A^{4} \leq \int_{-A}^{A} d \alpha \int_{V_{p, \alpha}} d \xi \int_{0}^{d(M)} W_{0}(t, \alpha) d t .
$$

From which we obtain the required bound.

Theorem 7.5. Let $\left(M^{3}, \Theta\right)$ be a complete, simply connected pseudohermitian 3manifold with non-positive Webster scalar curvature satisfying the torsion condition (1.1). Then given any domain $\Omega \subset M^{3}$ we have the following inequality:

$$
\operatorname{Vol}(\Omega) \leq C(\operatorname{Area}(\partial \Omega))^{4 / 3} .
$$

Lemma 7.6. Given any $T$-orbit $\Gamma$ in $M^{3}$, the exponential map defined by

$$
\exp _{\Gamma}(\xi)=\gamma(1)
$$

where $\gamma$ is the zero curvature geodesic with initial vector $\xi$, is a diffeomorphism.

Proof. The Jacobian determinant of $\exp _{\Gamma}$ is given by

$$
\Theta \wedge d \Theta\left(T, X, Y_{\phi}\right)=c_{\phi}^{\prime} .
$$

Under the curvature assumption, $c_{\phi}^{\prime}$ is bounded away from zero. Hence $\exp _{\Gamma}$ is a local diffeomorphism from $R^{3}$ onto its image. We claim the image is $M^{3}$. This follows from the fact that each point $q \in M$ can be joined to a point on the $T$-orbit by a length minimizing geodesic of curvature $\alpha$. If $\alpha$ is different from zero, we can deform this geodesic by a continuity argument to find a family of geodesics whose curvature decreases from $\alpha$ to zero. This follows from the implicit function theorem applied to the exponential map $\exp _{q}$, which is nonsingular at $(\xi, \alpha)$ and we also use the fact that the $T$-orbits are properly embedded. Thus $\exp _{\Gamma}$ is a covering map. But $M$ is simply connected and so $\exp _{\Gamma}$ is a diffeomorphism. 
Proof of Theorem 7.5. It follows from Lemma 7.6 that $\omega_{0}(p)=2 \pi$ for each point $p \in \Omega$

$$
\begin{aligned}
\operatorname{Vol}(\Omega) & =\operatorname{Vol}\left((U \Omega)_{0}\right) \\
& \leq \int_{\Lambda_{\partial \Omega}^{+}} \eta \cdot v d \sigma(\eta) \int_{0}^{l(\eta, 0)} d s \\
& \leq \int_{\partial \Omega} d A(x) \int_{0}^{\pi} l(\eta, 0) d \phi(\eta) .
\end{aligned}
$$

In order to bound the integral $\int_{0}^{\pi} l(\eta, 0) d \phi(\eta)$ we will compare it with the $p$-area of the $p$-minimal surface $\Sigma_{x}$ spanned by the zero curvature geodesics issuing from $x$ in the direction of inward pointing contact vector $\eta$. It is known that $\Sigma_{x}$ is a $p$ area minimizing surface [2]. We parametrize the vector $\eta$ by the angle $0 \leq \phi \leq \pi$ and the length parameter along the geodesic to $x$ by $l$, so that the surface $\Sigma_{x}$ is parametrized by the domain $D$ by the condition $0 \leq \phi \leq \pi, 0 \leq l \leq l(\phi)$. We also consider the subdomain $D^{\prime} \subset D$ described by the condition:0 $\leq \phi \leq \pi, l(\phi) / 2 \leq$ $l \leq l(\phi)$. It follows that $|D| \leq 2\left|D^{\prime}\right|$.

The area form on the minimal surface $\Sigma$ is given by [2]: $\Theta \wedge e^{1}$ in terms of the local framing $e_{1}$ which represents the contact unit tangent along $\Sigma$ so that $e_{1}, e_{2}=J e_{1}$, and $T$ gives a framing and $e^{1}, e^{2}, \Theta$ form the coframe field. Let $Y_{\phi}$ be the Jacobi vector field along the geodesic corresponding to varying the angle $\phi$, so that we find

$$
\Theta \wedge e^{1}\left(e_{1}, Y_{\phi}\right)=-c_{\phi},
$$

where $c_{\phi}$ satisfies the differential equation

$$
c_{\phi}^{\prime \prime \prime}+R c_{\phi}^{\prime}=0
$$

and the initial conditions $c_{\phi}(0)=c_{\phi}^{\prime}(0)=0$. It follows from a simple comparison that we may write:

$$
\begin{aligned}
\int_{0}^{\pi} l(\phi) d l & =|D| \leq 2\left|D^{\prime}\right| \leq \int_{D^{\prime}} d \phi d l \\
& \leq\left(\int_{D^{\prime}} l^{2} d \phi d l\right)^{1 / 3} \cdot\left(\int_{D^{\prime}} l^{-1} d \phi d l\right)^{2 / 3} \\
& \leq c_{0}\left(\int_{D^{\prime}} l^{2} d \phi d l\right)^{1 / 3} \\
& \leq c_{0} \operatorname{Area}\left(\Sigma_{x}\right)^{1 / 3}
\end{aligned}
$$

the last line follows because when $\alpha=0, c_{\phi}$, hei $(s)=s^{2}$, Lemma 2.1. From Lemma 3.1(a), $s^{2} \leq c_{\phi}(s)$ for $\alpha=0$, using the non-positivity of the Webster 
curvature. Hence $l^{2} \leq\left|\Theta \wedge e^{1}\left(e_{1}, Y_{\phi}\right)\right|$. Since $\Sigma_{x}$ is area minimizing relative to fixed boundary, it follows that $\operatorname{Area}\left(\Sigma_{x}\right) \leq \operatorname{Area}(\partial \Omega)$. Substituting this bound into the last inequality and integrating over $x \in \partial \Omega$ gives the desired inequality.

This argument, due to Pansu in the case of the Heisenberg group, exploits the special feature of minimal surfaces in 3D as surfaces ruled by contact geodesics, and hence easy to construct. In order to find an alternate argument that generalizes to situations in which the Webster curvature is positive, we generalize the argument of Croke to this setting.

Definition 7.7. For a $C^{1}$ domain $\Omega$ we say $w i d t h(\Omega) \geq w$ if each point $p \in \partial \Omega$ there is a ball of radius $w$ contained in $\Omega$ that is tangent to $\partial \Omega$ at $p$.

Theorem 7.8. Let $\left(M^{3}, \theta, J\right)$ be a compact pseudo-hermitian manifold satisfying the following:

(a) the torsion condition (1.1),

(b) the Webster scalar curvature satisfies $0 \leq R \leq C$,

(c) $\operatorname{diameter}\left(M^{3}\right) \leq D$,

(d) $\operatorname{Vol}\left(M^{3}\right) \geq V$;

then given any constant $w$, there exists an isoperimetric constant $C$ so that for any domain $\Omega \subset M$ of with width $(\Omega) \geq w$ and $\operatorname{Vol}(\Omega) \leq \operatorname{Vol}(M \backslash \Omega)$ the following holds:

$$
\operatorname{Vol}(\Omega) \leq C|\partial \Omega|^{4 / 3} .
$$

Proof. We begin with the formula valid for each $p \in \Omega$ :

$$
\operatorname{Vol}(\Omega)=\int_{S_{p}} d \phi(\xi) \int_{-\infty}^{\infty} d \alpha \int_{0}^{l(\xi, \alpha)} W(t, \xi, \alpha) d t .
$$

Now integrate this over $p \in \Omega$ :

$$
\begin{aligned}
\operatorname{Vol}^{2}(\Omega) & =\int_{\Omega} d V(p) \int_{S_{p}} d \phi(\xi) \int_{-\infty}^{\infty} d \alpha \int_{0}^{l(\xi, \alpha)} W(t, \xi, \alpha) d t \\
& =\int_{U(\Omega)} d \mu(\xi) \int_{-\infty}^{\infty} d \alpha \int_{0}^{l(\xi, \alpha)} W(t, \xi, \alpha) d t, \quad \text { by Lemma } 7.3 \\
& =\int_{-\infty}^{\infty} d \alpha \int_{\Lambda_{\alpha}^{+}(\partial \Omega)} \eta \cdot v d \sigma(\eta) \int_{0}^{l(\eta, \alpha)} d s \int_{0}^{l(\eta, \alpha)-s} W\left(t, \alpha,-\Psi_{s}(\eta)\right) d t .
\end{aligned}
$$


A brief calculation using the Wronskian bounds shows that the integral

$$
\int_{0}^{l} d s \int_{0}^{l-s} W(t, \alpha, \xi) d t \sim \begin{cases}l^{6} & \text { if } \alpha l \ll 1 \\ l^{2} / \alpha^{4} & \text { if } \alpha l \sim 1\end{cases}
$$

Hence,

$$
\begin{aligned}
\operatorname{Vol}^{2}(\Omega) & \geq C \int_{-\infty}^{\infty} d \alpha \int_{\Lambda_{\alpha}^{+}(\partial \Omega)} \eta \cdot v l(\eta, \alpha)^{6} d \sigma(\eta) \\
& \geq C \int_{-\infty}^{\infty}\left\{\int_{\Lambda_{\alpha}^{+}(\partial \Omega)} \eta \cdot \nu l(\eta, \alpha) d \sigma(\eta)\right\}^{6} d \alpha /(\operatorname{Area}(\partial \Omega))^{5} .
\end{aligned}
$$

We claim:

$$
\begin{array}{ll}
\operatorname{Vol}\left((U \Omega)_{\alpha}\right) \geq \omega_{\alpha} \operatorname{Vol}(\Omega) & \text { This is by definition. } \\
\operatorname{Vol}\left((U \Omega)_{\alpha}\right) \geq C_{A} \operatorname{Area}(\partial \Omega) & \text { for }|\alpha| \leq A_{0} \sim 1 / w .
\end{array}
$$

(7.6) follows from the assumption on width: we have

$$
\operatorname{Vol}\left((U \Omega)_{\alpha}\right)=\int_{S^{+}(\partial \Omega)} \eta \cdot v d \sigma \int_{0}^{l(\eta, \alpha)} W(t, \eta, \alpha) d t
$$

Since $l(\eta, \alpha) \geq w$ for $|\alpha| \leq A_{0} \sim 1 / w$, we have

$$
\begin{aligned}
\operatorname{Vol}\left((U \Omega)_{\alpha}\right) & \geq \int_{S^{+}(\partial \Omega)} \eta \cdot v d \sigma C\left(l_{0}, w\right) \\
& \geq C \operatorname{Area}(\partial \Omega)
\end{aligned}
$$

By the second identity in Lemma 7.3 with $f=1$,

$$
\int_{S^{+}(\partial \Omega)} \eta \cdot v d \sigma \int_{-A}^{A} l(\eta, \alpha)=\int_{-A}^{A} \operatorname{Vol}\left((U \Omega)_{\alpha}\right) d \alpha
$$

Using (7.5) we get,

$$
=\int_{-A}^{A} \int_{\Omega} \omega_{\alpha}(p) d V(p) d \alpha \geq\left(i n f_{p \in \Omega} \int_{-A}^{A} \omega_{\alpha}(p) d \alpha\right) \operatorname{Vol}(\Omega) .
$$


Apply Holder to the right hand side of (7.4):

$$
\operatorname{Vol}^{2}(\Omega) \geq\left(\int_{-A_{0}}^{A_{0}} \int_{S^{+}(\Omega)} \eta \cdot v l(\eta, \alpha) d \sigma d \alpha\right)^{6} /\left(2 A_{0} \operatorname{Area}(\partial \Omega)\right)^{5}
$$

by (7.6),

$$
\geq\left(\int_{-A_{0}}^{A_{0}} \operatorname{Vol}\left((U \Omega)_{\alpha}\right)\right)^{5} \cdot 2 A_{0} C_{A_{0}} \operatorname{Area}(\partial \Omega) /\left(2 A_{0} \operatorname{Area}(\partial \Omega)\right)^{5}
$$

by (7.7),

$$
\geq\left(\inf _{p \in \Omega} \int_{-A_{0}}^{A_{0}} \omega_{\alpha}(p) d \alpha\right)^{5} \operatorname{Vol}^{5}(\Omega) /\left(2 A_{0}\right)^{5} \operatorname{Area}^{4}(\partial \Omega)
$$

The estimate (7.3) then gives the required lower bound for $\inf _{p \in \Omega} \int_{-A_{0}}^{A_{0}} \omega_{\alpha}(p) d \alpha$ provided $A_{0}$ is chosen sufficiently large so that $C(D) / A_{0}{ }^{4} \leq \operatorname{Vol}(M) / 4$. This finishes the proof of the theorem.

Remark 7.9. In this argument, if we remove the width condition, it is still possible to obtain a general isoperimetric inequality of the form

$$
\operatorname{Vol}(\Omega) \leq C(\operatorname{Area}(\partial \Omega))^{5 / 4}
$$

This follows from the first line of (7.8), apply the bound $|\eta \cdot v| \leq 1$ and the Holder inequality.

\section{Morrey's lemma under geometric assumptions}

Definition 8.1. Let $\nabla_{b} f=\left(e_{1} f, e_{2} f\right)$.

Theorem 8.2. Assume that the Webster curvature is bounded and torsion vanishes. Then we have for $x \in B_{r}\left(x_{0}\right)$,

$$
\left|f(x)-\frac{1}{\operatorname{Vol}\left(B_{r}\left(x_{0}\right)\right)} \int_{B_{r}\left(x_{0}\right)} f(y) d y\right| \leq C \frac{r^{4}}{\operatorname{Vol}\left(B_{r}\left(x_{0}\right)\right)} \int_{B_{r}\left(x_{0}\right)} \frac{\left|\nabla_{b} f\right|}{d(x, z)^{3}} d z .
$$

Here $d(x, z)$ denotes the metric distance from $x$ to $z$. The constant $C$ only depends on the bounds for the curvature. 
Proof. We easily bound the left side by,

$$
\frac{1}{\operatorname{Vol}\left(B_{r}\left(x_{0}\right)\right)} \int_{B_{r}\left(x_{0}\right)}|f(y)-f(x)| d y .
$$

Now for fixed $x$, connect $x$ to $y$ by a minimizing geodesic parametrized so that $\gamma(0)=x, \gamma(1)=y$. Such a geodesic is obtained from a unit speed geodesic $\tilde{\gamma}(t)$, by setting,

$$
\gamma(t)=\tilde{\gamma}(t d(x, y))
$$

Thus $\left|\gamma^{\prime}(t)\right| \leq d(x, y)$. Now,

$$
|f(x)-f(y)| \leq d(x, y) \int_{0}^{1}\left|\nabla_{b} f(\gamma(t, x, y))\right| d t .
$$

The last inequality when substituted in (8.1) yields,

$$
\frac{1}{\operatorname{Vol}\left(B_{r}\left(x_{0}\right)\right)} \int_{B_{r}\left(x_{0}\right)} \int_{0}^{1}\left|\nabla_{b} f(\gamma(t, x, y))\right| d(x, y) d t d y .
$$

Now in (8.2) we make a change of variable. We set, $\gamma(t, x, y)=z$. Note $x$ is fixed and so is $t$. We now need to compute the Jacobian change by this change of variable. That is we move the end-point $y$ of our geodesic through geodesic (Legendrian) variation and see what happens to the Jacobian density at a fixed $t$ along the curve. That is nothing else but our Wronskian formula which we will proceed to do. Now observe if $\gamma(t, x, y)=z$, then $t d(x, y)=d(x, z)$, by the nature of our normalizations. So we have,

$$
t d(x, y)=d(x, z)
$$

Using (8.3) in (8.2) we get, (8.2) is bounded by,

$$
\frac{1}{\operatorname{Vol}\left(B_{r}\left(x_{)}\right)\right.} \int_{B_{r}\left(x_{0}\right)} \int_{0}^{1}\left|\nabla_{b} f(z)\right| d(x, z) \Gamma(y, \phi, z, t) \frac{d t}{t} d z .
$$

We now proceed to compute the Jacobian change in density $\Gamma$. Recall $x$ is fixed, so introducing polar coordinates centered at $x$, it easy to check that,

$$
\Gamma(y, \phi, z, t)=\frac{W(x, 1, \phi)}{W(x, t, \phi, z)} .
$$

where $W(x, \phi, 1)$ is our Wronskian when we have reached $t=1$, that is at $y$ and the denominator is our Wronskian when we are at $t$ that is at $z$. Note in (8.5) the ratio has a uniform bound irrespective of the speed at which we are running the geodesics, and in fact, we easily compute from our curvature bounds( we need 
both upper and lower bounds, one for the numerator Wronskian and one for the denominator Wronskian), that,

$$
\Gamma(y, \phi, z, t) \leq \frac{C}{t^{4}} .
$$

Notice that the bounds are obtained by comparison with the spaces of constant curvature, and on spaces of constant curvature the Wronskian is indeed $\phi$ independent and so the right side of the bound in (8.6) on the ratio of the Wronskians is indeed independent of $\phi$.

From (8.3) we also have since $d(x, y) \leq r$, that for a fixed $z$,

$$
t \geq \frac{d(x, z)}{r} .
$$

Using (8.6) and (8.7) the integral (8.4) is bounded by,

$$
\frac{1}{\operatorname{Vol}\left(B_{r}\left(x_{0}\right)\right)} \int_{B_{r}\left(x_{0}\right)} \int_{\frac{d(x, z)}{r}}^{\infty}\left|\nabla_{b} f(z)\right| d(x, z) \frac{d t}{t^{5}} d z .
$$

Performing the $t$ integration we get our theorem.

The Morrey lemma follows by applying Holder to the right side of Theorem 7.5 If we apply Holder with exponents, $p=4+\epsilon$ and $q, q=p /(p-1)$, we see that,

$$
\left|f(x)-\frac{1}{\operatorname{Vol}\left(B_{r}\left(x_{0}\right)\right)} \int_{B_{r}\left(x_{0}\right)} f(y) d y\right| \leq C r^{(4-3 q) / q} .
$$

Thus if $x, w$ are such that $d(x, w)=r$, we may apply the result to a ball centered at $w$ of radius $r$, to conclude,

Theorem 8.3. Assume $\left|\nabla_{b} f\right| \in L^{p}$, with $p>4$. then,

$$
|f(x)-f(w)| \leq C r^{(4-3 q) / q} .
$$

\section{References}

[1] A. Bellaiche, The tangent space in Sub-Riemannian Geometry, in Sub-Riemannian Geometry, Progr. Math., Birkhäuser, Basel 144 (1996), 1-78.

[2] J.-H. Cheng, J.-F. Hwang, A. Malchiodi and P. Yang, Minimal surfaces in pseudohermitian geometry, Ann. Scuola Norm. Sup. Pisa Cl. Sci. 4 (2005), 129-177.

[3] C. Croke, Some isoperimetric inequalities and eigenvalue estimates, Ann. Sci. Ecole Norm. Sup. 13 (1980), 419-435.

[4] S. S. ChERN and R. S. HAMilton, On Riemannian metrics adapted to three dimensional contact manifolds, with an appendix by A. Weinstein, Lecture Notes in Math., Vol. 1111, Springer Berlin, 1985, 279-308. 
[5] P. PAnsu Une inegalite isoperimetrique sur le group de Heisenberg, C.R. Acad. Sc. Paris 295 (1982), 127-130.

[6] M. Rumin, Forms differentielles sur les varietés de contact, J. Differential Geom. 39 (1994), 281-330.

[7] N. TanaKa, "A Differential Geometric Study on Strongly Pseudo-convex Manifolds", Kinokuniya, Tokyo, 1975.

[8] F. TREves "Introduction to Pseudo-differential and Fourier Integral Operators", Vol. 1, Plenum Press.

[9] N. VAROPOUlos; Sobolev inequalities on Lie groups and symmetric spaces, J. Funct. Anal. 86 (1989), 19-40.

[10] S. Webster, Pseudo-Hermitian structures on a real hypersurface, J. Differential Geom. 13 (1978), 25-41.

Department of Mathematics Rutgers University

110 Frelinghuysen Rd.

Piscataway, NJ 08854

chanillo@math.rutgers.edu

Department of Mathematics

Princeton University

Princeton, NJ 08544

yang@math.princeton.edu 